\title{
Effect of miscentering and low-dose protocols on contrast resolution in computed tomography head examination
}

\begin{abstract}
Background: Unoptimized protocols, including a miscentered position, might affect the outcome of diagnostic in CT examinations. In this study, we investigate the effects of miscentering position during CT head examination on the signal-to-noise ratio (SNR) and contrast-to-noise ratio (CNR). Method: We simulate the CT head examination using a water phantom with a standard protocol $(120 \mathrm{kVp} / 180 \mathrm{mAs})$ and a low dose protocol $(100 \mathrm{kVp} / 142 \mathrm{mAs})$. The table height was adjusted to simulate miscentering by $5 \mathrm{~cm}$ from the isocenter, where the height was miscentered superiorly (MCS) at 109, 114, 119, and $124 \mathrm{~cm}$, and miscentered inferiorly (MCI) at 99, 94, 89, and $84 \mathrm{~cm}$. Seven circular regions of interest were used, with one drawn at the center, four at the peripheral area of the phantom, and two at the background area of the image. Results: For the standard protocol, the mean CNR decreased uniformly as table height increased and significantly differed $(\mathrm{p}<0.05)$ at $+20 \mathrm{~cm}$ for MCS $(435.70 \pm 9.39)$ and $-20 \mathrm{~cm}$ for MCI $(438.91 \pm 10.94)$ from the isocenter. Similarly, significant reductions $(\mathrm{p}<0.05)$ were also noted for SNR for MCS (at +20 $\mathrm{cm}$ ) and MCI (at $-20 \mathrm{~cm}$ ). For the low dose protocol, both CNR and SNR were significantly reduced $(\mathrm{p}<0.05)$ at table heights of +20 and $-20 \mathrm{~cm}$ from the isocenter. Conclusion: Miscentering is proven to significantly affect the image quality in both low and standard dose protocols for head CT procedure. This study implies that accurate patient centering is one of the approaches that can improve CT optimization practice.
\end{abstract}

Keyword: CT head; Miscentered position; Image quality; SNRCNR 